\title{
Establishment and Characterization of a Telomerase-Immortalized Sheep Trophoblast Cell Line
}

\author{
Yufei Zhang, ${ }^{1}$ Jing Shi, ${ }^{1}$ and Shuying Liu ${ }^{1,2}$ \\ ${ }^{1}$ College of Veterinary Medicine, Inner Mongolia Agricultural University, Hohhot 010018, China \\ ${ }^{2}$ Key Laboratory of Clinical Diagnosis and Treatment Technology in Animal Disease, Ministry of Agriculture, Hohhot 010018, China \\ Correspondence should be addressed to Shuying Liu; liushuying_imau@126.com
}

Received 12 September 2015; Revised 16 December 2015; Accepted 13 January 2016

Academic Editor: Louiza Belkacemi

Copyright (c) 2016 Yufei Zhang et al. This is an open access article distributed under the Creative Commons Attribution License, which permits unrestricted use, distribution, and reproduction in any medium, provided the original work is properly cited.

\begin{abstract}
The primary sheep trophoblast cells (STCs) have a finite lifespan in culture. This feature limits the scope for long-term in vitro studies with STCs. This study was an attempt to establish and characterize a telomerase-immortalized sheep trophoblast cell line. STCs were isolated and purified by using Percoll and specific immunoaffinity purification, respectively. The purified STCs were transfected with a plasmid carrying sequences of human telomerase reverse transcriptase (hTERT) to create immortalized sheep trophoblast cell line (hTERT-STCs). hTERT-STCs showed a stable expression of hTERT gene, serially passaged for a year, and showed active proliferation without signs of senescence. Cytokeratin 7 (CK-7), secreted human chorionic gonadotrophin subunit $\beta(\mathrm{CG}-\beta)$, placental lactogen (PL), and endogenous jaagsiekte sheep retrovirus (enJSRV) envelope genes were expressed in hTERTSTCs. Transwell cell invasion assay indicated that hTERT-STCs still possessed the same invasive characteristics as normal primary sheep trophoblast cells. hTERT-STCs could not grow in soft agar and did not develop into tumors in nude mice. In this study, we established a strain of immortalized sheep trophoblast cell line which could be gainfully employed in the future as an experimental model to study trophoblast cells with secretory function, invasive features, and probable biological function of enJSRV envelope genes.
\end{abstract}

\section{Introduction}

Mammalian placentae are unique organs serving as the interface between the fetal and maternal tissues. Although placentae of different animals are dissimilar in shapes, sizes, and organizational structure, all share the same functionality; for example, the placentae have a fundamental role in the exchange of nutrients, ions, water, respiratory gases, vitamins, nutrients, and other molecules which are necessary for fetal metabolism and development. In addition, the placenta produces hormones and also acts as a barrier that protects the fetus from insult from the maternal immune system [1].

The functional cell type of the placenta is the trophoblast cell. There are some differences between the type and role of trophoblast cells among different species. Human placental trophoblasts include cytotrophoblast (CTB), syncytiotrophoblast (STB), and extravillous trophoblast (EVT) [2]. The multinucleated syncytiotrophoblasts covered on the surface of villi are formed by differentiation and fusion of cytotrophoblasts $[3,4]$. The multinucleated syncytiotrophoblasts secrete hormones such as human chorionic gonadotrophin (HCG) and placental prolactin. These hormones enter the maternal circulation and play an important role in the maintenance of pregnancy and immune response $[4,5]$. Extravillous cytotrophoblasts exhibit strong extended invasiveness and erode and invade the endometrium until maternal spiral arteries are able to anchor the conceptus in uterus, while the CTB represents the medium for the nutrient and gas exchange vital for the growth of the fetus $[6,7]$. Histologically, the sheep placenta is classified as cotyledonary placenta or multiplex type of placenta. Trophoblast cells differentiate into four subsets, that is, mononuclear trophoblast cells (MTCs), binucleate cells (BNCs), trinucleate cells (TNCs), and multinucleated syncytiotrophoblasts $[6,8]$. The BNCs differentiate from the MTC through mitosis and polyploidy formation which involves consecutive nuclear divisions without 
cytokinesis $[9,10]$. Some of the BNCs merge with the endometrial epithelial cells of the uterus and evolve as TNCs. Other BNCs fuse with the trinucleate cells to form multinucleated syncytial plaques with 20-25 nuclei [9]. Cotyledons, the specialized structure formed between the multinucleated syncytial plaques and BNC, take shape as placentome with maternal endometrium through reciprocal chiasmata $[9,11]$. Hemotrophic nutrition is passed through the placentomes to the fetus [9]. Other functions of multinucleated syncytial plaques and $\mathrm{BNC}$ include the production of important proteins and hormones, such as placental prolactin, chorionic gonadotrophin, pregnancy associated glycoprotein, and progesterone [9].

The trophoblast cell lesions are often involved in the causation of obstetric disorders including fetal growth restriction, preeclampsia, and even abortion $[6,12,13]$. Therefore, trophoblast cell lines generated from normal cells represent an invaluable vehicle for research on trophoblast cell biology, immunology, endocrine function, placental development, and mechanisms of infectious and noninfectious diseases [14]. However, the isolated primary trophoblast cells rapidly lose the ability to proliferate, to differentiate, and to secrete hormones, which greatly limits the scope of its use in in vitro studies. Establishment of a cell line that can overcome this limitation is a key research imperative.

Cultured normal human cells have a finite lifespan due to replicative senescence, which is associated with progressive shortening of cell telomeres $[6,15,16]$. Telomeres found at the ends of chromosomes in eukaryotes have been shown to protect the chromosome ends and maintain cell immortality $[16,17]$. By introducing exogenous telomerase reverse transcriptase (hTERT) gene, cells appeared to acquire the ability for unlimited proliferation through the activation of telomerase $[18,19]$. Studies have shown that the introduction of hTERT gene enables establishment of immortalized cell line which retains the original characteristics of the normal cells $[6,20,21]$.

In this study, we sought to establish a stable sheep trophoblast cell line expressing exogenous hTERT gene and profiled its phenotype and functionality.

\section{Materials and Methods}

2.1. Isolation, Purification, and Culture of Sheep Trophoblast Cells. Pregnant Mongolian sheep uteri (45-60 days of pregnancy) provided by the Hohhot slaughterhouse were immediately transferred to the laboratory in a thermal container with a heat preservation vessel containing sterilized saline at $37^{\circ} \mathrm{C}$. The phase of pregnancy was estimated by measuring the fetal crown rump length [6]. The primary sheep trophoblast cells (STCs) were separated from the tissue samples and cultured as described by Petroff et al. [22] with some modifications. In brief, the uterus was cleaned with $70 \%$ ethanol and dissected in the sterile console, and the cotyledon was mechanically separated with tweezers and placed in a sterile Petri dish $10 \mathrm{~cm}$ in diameter. The cotyledons were meticulously minced and dissociated in $100 \mathrm{~mL}$ Hank's balanced salt solution (HBSS) with $25 \mathrm{mmol}$ HEPES, $0.2 \mathrm{mg} / \mathrm{mL}$ DNaseI (Sigma, St. Louis, MO, USA), and 0.25\% trypsin (Invitrogen,
Carlsbad, CA, USA) for $30 \mathrm{~min}$ at $37^{\circ} \mathrm{C}$ in a rotating waterbath shaker $(150 \mathrm{rpm})$. The dispersed cells were isolated by $200 \mu \mathrm{m}$ mesh stainless steel screens and kept in $15 \%$ fetal bovine serum (FBS; Gibco, Grand Island, NY, USA). The filtrate was centrifuged at $1000 \mathrm{~g}$ for $10 \mathrm{~min}$ and resuspended in serum-free DMEM/F12 medium (Gibco, Grand Island, NY, USA). The cell suspension was transferred to a test tube containing 30\% and 50\% Percoll gradient and centrifuged continuously with a swinging bucket rotor at $1200 \mathrm{~g}$ for $20 \mathrm{~min}$ at room temperature (RT). The upper diffuse band containing the STCs was aspirated with Pasteur pipette. The cells were washed by a fourfold volume of nonserum culture medium and centrifuged at $1000 \mathrm{~g}$ for $5 \mathrm{~min}$ and suspended with $1 \mathrm{~mL}$ cell separation buffer (0.5\% BSA, 0.08\% EDTA $\mathrm{pH}$ 7.2, PBS, and vacuum filtration sterilization as well as liquid within gas). Cells $\left(2 \times 10^{8}\right.$ cells $\left./ \mathrm{mL}\right)$ were incubated with $40-60 \mu \mathrm{g} / \mathrm{mL}$ anti-human HLA-ABC (Miltenyi Biotec, Bergisch Gladbach, Germany) for $30 \mathrm{~min}$ at $4^{\circ} \mathrm{C}$. The cells were incubated with goat anti-mouse IgG microbeads (Miltenyi Biotec, Bergisch Gladbach, Germany) and then purified by immune negative selection. After immunodepletion, the cells were centrifuged at $400 \mathrm{~g}$ and resuspended in complete DMEM/F12 medium within 15\% fetal bovine serum. The purified cells were plated onto applicable culture vessels with culture medium at a minimum of $2 \times 10^{5} \mathrm{cells} / \mathrm{cm}^{2}$ and incubated in an atmosphere of $5 \% \mathrm{CO}_{2}$ at $37^{\circ} \mathrm{C}$.

2.2. Transfections and Establishment of Cell Line. The primary sheep trophoblast cells were treated with $0.25 \%$ trypsin to obtain the single cells. The cells were then washed with preheated Opti-MEM Medium twice, and their density was adjusted to $1 \times 10^{7} / \mathrm{mL}$. After a gentle mix, the mixture of $100 \mu \mathrm{L}$ cell suspension (approximately $1 \times 10^{6}$ cells) and $10 \mu \mathrm{g}$ pCI-neo-hTERT plasmid DNA was placed in $2 \mathrm{~mm}$ gap electroporation cuvettes (BTX, Holliston, MA, USA) and subjected to a pulse of $150 \mathrm{~V}$ and $5 \mathrm{~ms}$ pulse length by an electroporator (NEPA21; Tokiwa Science, Tokyo) and then seeded into preheated $100 \mathrm{~mm}$ dish containing $15 \mathrm{~mL}$ complete DMEM/F12 medium. The transfected cells were plated in medium containing $500 \mu \mathrm{g} / \mathrm{mL}$ G418 (Sigma, St. Louis, MO, USA) for two to three weeks $48 \mathrm{~h}$ after transfection. The hTERT-transfected sheep trophoblast cell (hTERTSTCs) clones were cultured in complete DMEM/F12 medium supplemented with EGF (Sigma, St. Louis, MO, USA) and $300 \mu \mathrm{g} / \mathrm{mL}$ of $\mathrm{G} 418$ [6].

2.3. Reverse Transcription Polymerase Chain Reaction for cDNA Synthesis. Total RNA from STCs and hTERTSTCs was isolated by using RNAprep Pure Micro Kit (TIANGEN, Beijing, China) according to the manufacturer's instructions. Reverse transcriptase polymerase chain reaction (RT-PCR) was performed using One-Step RT-PCR Kit method (TaKaRa, Dalian, China). The primers of hTERT, GAPDH, endogenous jaagsiekte sheep retrovirus envelope gene (enJSRV-env), and syncytin-Ruml were designed by mRNA sequences of [NM_198253.2], [NM_001190390.1], [AF153615], and [JX412969.1] to obtain the respective cDNAs. PCR was performed using particular conditions for 
TABLE 1: Primers and conditions used in RT-PCR gene expression.

\begin{tabular}{|c|c|c|c|c|}
\hline Gene & Sequence $\left(5^{\prime}-3^{\prime}\right)$ & Cycles & Product size (bp) & Anneal. temp. ${ }^{\circ} \mathrm{C}$ \\
\hline \multicolumn{5}{|l|}{ hTERT } \\
\hline Sense & CGTACATGCGACAGTTCGTG & 35 & 408 & 55 \\
\hline Antisense & AGTTCACCACTGTCTTCCGC & & & \\
\hline \multicolumn{5}{|l|}{ enJSRV-env } \\
\hline Sense & ATGCCGAAGCGCCGCGCTGGATT & 35 & 1836 & 60 \\
\hline Antisense & TCACGGGTCGTCCCCCGCAGCTC & & & \\
\hline \multicolumn{5}{|c|}{ syncytin-Rum1 } \\
\hline Sense & GATGGAGCTGGGTAAGCGAC & 35 & 2014 & 60 \\
\hline Antisense & TTGTAGCCACGAGTTCCAGG & & & \\
\hline \multicolumn{5}{|l|}{ GAPDH } \\
\hline Sense & GTTTGTGATGGGCGTGAACC & 35 & 300 & 55 \\
\hline Antisense & CCAGTGAGCTTCCCGTTGAG & & & \\
\hline
\end{tabular}

RT-PCR: reverse transcriptase polymerase chain reaction; enJSRV-env: endogenous jaagsiekte sheep retrovirus envelope gene; and hTERT: human telomerase reverse transcriptase.

the following genes: hTERT, GAPDH, enJSRV-env, and syncytin-Rum1. The PCR conditions used during reactions are mentioned in Table 1. Following the PCR reaction, products were electrophoresed by $1 \%$ agarose gel electrophoresis and stained with ethidium bromide.

2.4. Western Blot Analysis. The day before transfection, $1.0 \times$ $10^{5}$ cells of primary STCs and hTERT-STCs were, respectively, plated in $60 \mathrm{~mm}$ Petri dish and total proteins were extracted from $24 \mathrm{~h}$ culture by using the M-PER Mammalian Kit (Thermo Fisher Scientific, Beijing, China). Protein samples were normalized with Bradford reagent (Bio-Rad, Beijing, China). After separation by SDS-PAGE electrophoresis, proteins were transferred to a PVDF membrane using the semidry method with constant voltage of $25 \mathrm{~V}$ for $15 \mathrm{~min}$. The membrane was blocked with 5\% nonfat dry milk for $1 \mathrm{~h}$ at $\mathrm{RT}$ and then incubated with rabbit polyclonal anti-telomerase reverse transcriptase (1:500; Abcam, Cambridge, MA, USA), overnight at $4^{\circ} \mathrm{C}$. Blots were washed in TBST thrice for $5 \mathrm{~min}$ each. The membrane was then incubated with goat antirabbit secondary antibody with HRP conjugation $(1: 2000$; Abcam, Cambridge, MA, USA) for $2 \mathrm{~h}$ at $37^{\circ} \mathrm{C}$. After three washes with TBST, the membrane blots were detected by chemiluminescence. GAPDH were used as internal controls.

2.5. Immunofluorescence. $5 \times 10^{3}$ cells were seeded onto chamber slides until $50 \%$ confluency was achieved. They were washed with PBS and fixed with $4 \%$ paraformaldehyde at RT for 15 min. After permeabilization with $0.1 \%$ Triton X-100 in PBS for 5 min at RT, the cells were blocked with $10 \%$ normal goat serum for $1 \mathrm{~h}$. The cells were incubated with rabbit monoclonal anti-cytokeratin 7 (CK-7) antibodies (1:200; Thermo Fisher Scientific, Beijing, China) containing 10\% normal goat serum for $14 \mathrm{~h}$ at $4^{\circ} \mathrm{C}$. With a subsequent wash, the cells were incubated with goat anti-rabbit-Texas Red labelled antibodies (1:100; Thermo Fisher Scientific, Beijing, China) for $1 \mathrm{~h}$ at $37^{\circ} \mathrm{C}$ and were mounted with cover slips and observed under a Zeiss Axio Observer microscope (Carl
Zeiss, Oberkochen, Germany) after staining with $0.5 \mu \mathrm{g} / \mathrm{mL}$ 4-6-diamidino-2-phenylindole (DAPI) for $10 \mathrm{~min}$.

2.6. Hormone Assay. The assay method described by Dong et al. was used for assessing the secretion of chorionic gonadotrophin and placental lactogen [6].

2.7. Cell Invasion Assay. Transwell membranes $(8.0 \mu \mathrm{m}$ pore size, Coring, NY, USA) coated with Matrigel on upper and bottom chambers were dried at $4^{\circ} \mathrm{C}$ for $1 \mathrm{~h}$. The upper chamber was incubated with $300 \mu \mathrm{L}$ of preheated serumfree medium for $30 \mathrm{~min}$ at RT for matrix rehydration. Primary STCs and hTERT-STCs $\left(3 \times 10^{5}\right)$ were plated in the upper chamber in serum-free Opti-MEM I Medium, respectively. The lower chambers were filled with $500 \mu \mathrm{L}$ complete DMEM/F12 medium. Following an incubation for $48 \mathrm{~h}$, the culture medium was discarded and washed with PBS. The cells on Matrigel and upper chamber were wiped with a cotton swab. The migrated cells were fixed with $90 \%$ ethanol for $30 \mathrm{~min}$ at RT, stained with Giemsa, and examined under a light microscope.

2.8. Soft Agar Assay and Test of Tumorigenicity. Six-well plates were filled with two $\mathrm{mL}$ of $0.66 \%$ noble agar as a bottom layer. Each of the 3000 primary STCs and hTERT-STCs cells, respectively, was blended with $0.35 \%$ soft agar and plated onto wells with $5 \% \mathrm{CO}_{2}$ and cultured at $37^{\circ} \mathrm{C}$ for 14 days. HeLa cells served as positive control, and the results were observed under an inverted microscope.

Cells in the logarithmic growth phase were collected and subjected to trypsinization, washed with PBS thrice, and suspended in a serum-free medium and the cell concentration was adjusted to $1 \times 10^{7} / \mathrm{mL}$ by cell counting. $0.2 \mathrm{~mL}$ cell suspension was subcutaneously injected into the right flanks of nude mice. After two months, the mice were decapitated and dissected for subcutaneous tumor nodules. These were paraffin embedded and subjected to hematoxylin and eosin (H\&E) staining. 


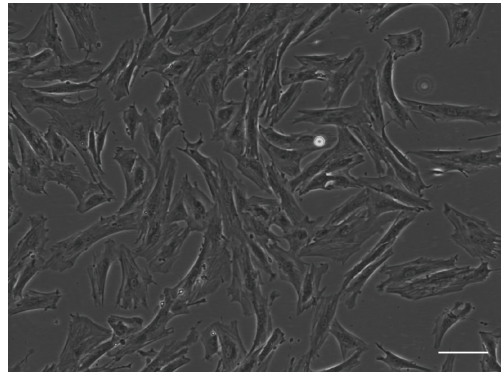

(a)

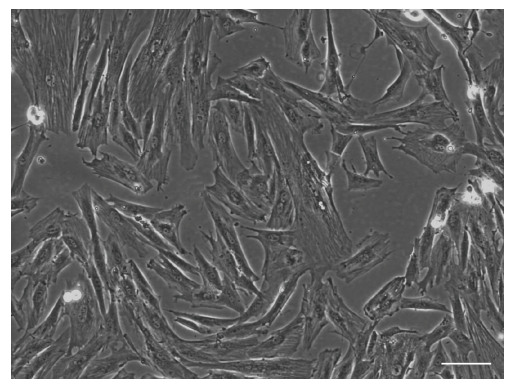

(c)

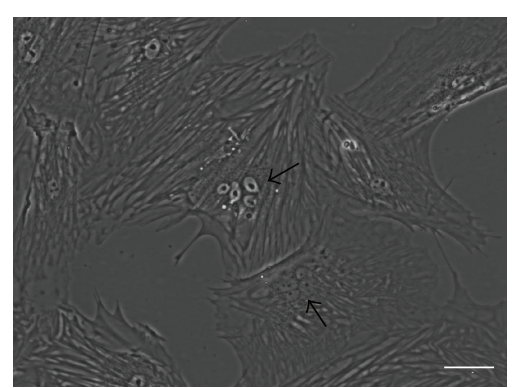

(b)

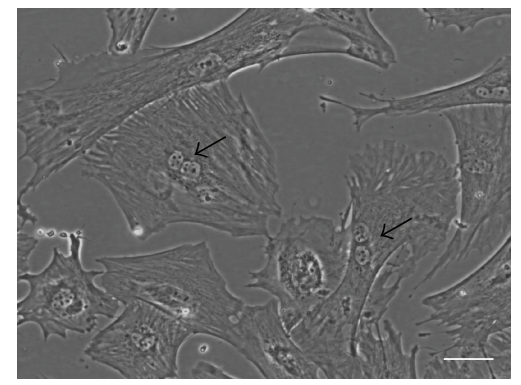

(d)

FIGURE 1: Primary sheep trophoblast cells and immortalized sheep trophoblast cells under phase contrast microscopy. (a) Primary STCs at passage 2; (b) multinucleated syncytiotrophoblast from primary STCs; (c) hTERT-STCs at passage 50; and (d) binucleate trophoblast cells from hTERT-STCs (scale bars, $50 \mu \mathrm{m}$ ). hTERT-STCs: human telomerase reverse transcriptase-sheep trophoblast cell line.

2.9. Statistical Tests. Data are presented as mean \pm standard deviation (SD). Statistical analyses were conducted using SAS 8.1 (SAS Institute, Cary, NC, USA) to determine intergroup differences in secretion of hormones; $P<0.05$ was considered statistically significant.

\section{Results}

3.1. Morphological Characteristics of STCs and hTERT-STCs. The primary sheep trophoblast cells (STCs) obtained from pregnant Mongolian sheep (45-60 days of pregnancy) were mainly mononuclear cells that showed epithelial celllike growth and morphological diversity, with oval nuclei (Figure 1(a)). On subculture of cells, intercellular fusion formed binucleate trophoblast cells, multinucleated syncytium (Figure 1(b)). After trypsinization and subculturing of sheep trophoblast cells, adherent growth was observable within $4 \mathrm{~h}$. However, with the increase of trophoblast cell passage number, cell proliferation was visibly decreased and had stopped growing by the 7th generation, with a large number of cells dead on account of senescence.

The STCs were transfected with pCI-neo plasmid containing the cDNA for hTERT for conversion to an immortalized sheep trophoblast cell line referred to as hTERT-STCs. The morphology of hTERT-STCs cells remained similar to primary STCs during one year of cultivation (Figure 1(c)). hTERT-STCs could also form binucleate trophoblast cells and multinucleated syncytium through intercellular fusion. Due to lesser number of multinucleated syncytium cells, we gave priority to binucleate trophoblast cells (Figure 1(d)).
3.2. hTERT Expression in hTERT-STCs. Total RNA and total protein of primary STCs, 30th and 50th generation hTERTSTCs cells, and HeLa cells were extracted and subjected to RT-PCR and Western blot analysis. PCR product was confirmed by $1 \%$ agarose gel electrophoresis analysis. The results showed that the total RNA of hTERT-STCs and HeLa cells showed expression of amplified $400 \mathrm{bp}$ hTERT band, while no such band was observed in the primary STCs (Figure 2(a)). This suggests that the hTERT-STCs cells had a stable expression of the mRNA of hTERT gene. Similar results were obtained by Western blot assay, where the hTERT protein $(120 \mathrm{kD})$ was expressed in hTERT-STCs and HeLa cells, but not in primary STCs (Figure 2(b)). These results indicate that the immortalized hTERT-STCs obtained by this method retained the ability to proliferate in vitro and were amenable to culture in the longer term.

3.3. Phenotypic Characteristics of hTERT-STCs. The most widely used phenotypic markers of trophoblast cell lines are cytokeratins, placental lactogen (PL), chorionic gonadotrophin (CG), and leukocyte antigen class I molecules [14]. The methods described in this research summarize the enzymatic dispersion, density gradient centrifugation, and leukocyte antigen class I (LAC-I) depletion of trophoblast cells. There are other placental cell types which express LACI molecules, except for the trophoblast cells. Thus, using the latter method to deplete these types of hybrid cells, we obtained cells which did not express the LAC-I molecules. Immunofluorescence staining for cytokeratin 7 showed that 


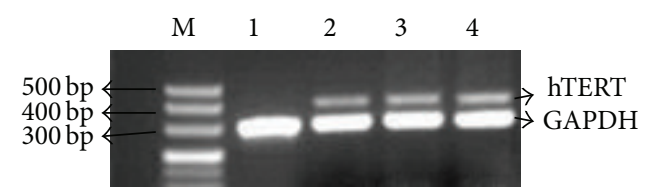

(a)

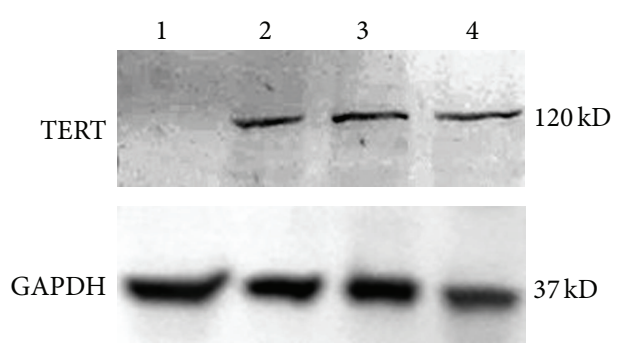

(b)

FIGURE 2: Retention of telomerase expression in hTERT-STCs. (a) Comparison of hTERT gene expression between hTERT-STCs and STCs by RT-PCR. M was the DL 500 DNA makers. Lane 1 was primary STCs; lane 2 was hTERT-STCs at passage 20; lane 3 was hTERT-STCs at passage 50; and lane 4 was HeLa cells (positive control). (b) Comparison of hTERT protein expression between hTERT-STCs and STCs by Western blot. Lanes 1-4 are the same as mentioned in (a). hTERT-STCs: human telomerase reverse transcriptase-sheep trophoblast cell line. RT-PCR: reverse transcriptase polymerase chain reaction.

the primary STCs purity was $>97 \%$ (Figure 3(a) (1)) and $100 \%$ pure hTERT-STCs (Figure 3(a) (2)).

The hormone analysis of cell culture revealed that both primary STCs and hTERT-STCs secreted CG- $\beta$ and PL, with no significant difference in secretory levels of these hormones $(P>0.05)$ (Figures 3(b) and 3(c)). In the sheep placenta, enJSRV-env is expressed in the mononuclear trophectoderm cells of the conceptus and is abundant in the trophoblast giant binucleated cells (BNCs) and multinucleated syncytial plaques of the placentomes [23-25].

The RT-PCR for enJSRV-env and syncytin-Ruml showed that enJSRV-env was detected in the primary STCs and hTERT-STCs, but syncytin-Ruml was detected only in the sheep genome (Figure 3(d)).

3.4. In Vitro Invasion Assay and Proliferation Analysis of Primary STCs and hTERT-STCs. The invasive capacity of trophoblast cells is an essential prerequisite for the development of mammalian placenta. We studied the invasive characteristics of primary STCs and hTERT-STCs in vitro by Transwell invasion assay; the results showed that both primary STCs (Figure 4(a)) and hTERT-STCs (Figure 4(b)) exhibited invasive ability, indicating that the latter had inherited the invasive ability of the former. HeLa cells (Figure 4(c)) served as a positive control.

Cell counting method was used to test the proliferation ability of primary STCs and hTERT-STCs cells, and the results when plotted as curves (Figure $4(\mathrm{~d})$ ) demonstrated two different kinds of cell proliferation patterns, which tended to increase with time. No significant difference was observed between the proliferation abilities of primary STCs and hTERT-STCs $(P>0.05)$.

3.5. Nonmalignant Transformation of hTERT-STCs In Vitro and In Vivo. Soft agar-based clonogenic assays are commonly employed to detect transformed cells. Soft agar-based clonogenic assays were used to detect malignant transformation of hTERT-STCs. We observed that after 14 days of incubation hTERT-STCs could not form colonies (Figure 5(a)), as compared to positive control HeLa (Figure 5(b)), which formed clones greater than $100 \mu \mathrm{m}$ diameter. This suggests that the hTERT-STCs had not acquired the ability of anchorageindependent growth.

The tumorigenicity of the immortalized sheep trophoblast cell line was then studied in vivo by subcutaneous injection of $1 \times 10^{6}$ hTERT-STCs cells in the flank of nude mice. No evidence of tumor formation was observed in these mice after 2 months. Histological examination revealed normal loose connective tissue below the injection sites and abnormally hyperplastic cells (Figure 5(c)). Nude mice injected with HeLa cells developed a localized mass at 3 weeks, which gradually increased, with a large number of abnormally proliferating tumor cells as evidenced by histological dysplasia (Figure 5(d)). These data indicate that the hTERT-STCs had become immortal cells but had acquired no other transformation characteristics, rendering them virtually akin to normal cells.

\section{Discussion}

Placenta is composed of trophoblast cells, macrophages, fibroblasts, stromal cells, mesenchymal stem cells, and some other varieties of cells. Among these, the trophoblast cells are the most common ones. Despite some shortcomings, enzyme digestion and tissue block are the most commonly used methods for isolation of trophoblast cells for primary culture. In this study, we used $30 \%$ and $50 \%$ concentration of Percoll to separate trophoblast cells, which removed a large number of blood cells, and some tissue debris fibroblasts. Finally, we used MACS filter to remove fibroblast cells, stromal cells, and mesenchymal stem cells. The immunofluorescence of purified sheep trophoblast cells revealed that $97 \%$ of the cells expressed specific marker such as CK7. This implied that the purified ovine trophoblast cells could be used for further studies.

Primary STCs retain most of their physical characteristics in vivo, thus mirroring the status and functionality of these cells under normal physiological conditions, making them well suited for use in studies on cell biology. However, the number of each separation and the culture time are limited, and the STCs last for about seven generations before stoppage of proliferation. The requirement for repeated separation and 

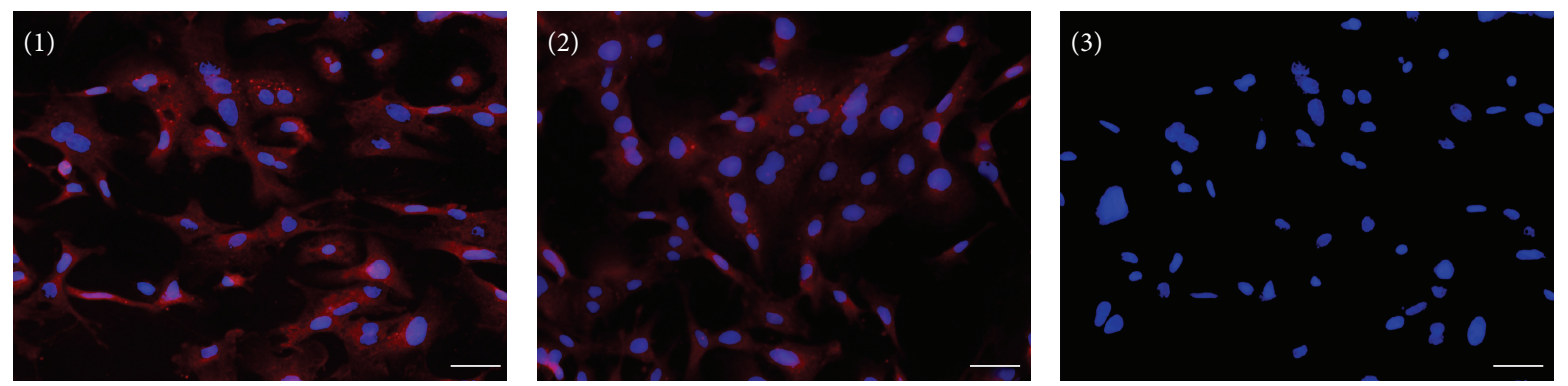

(a)

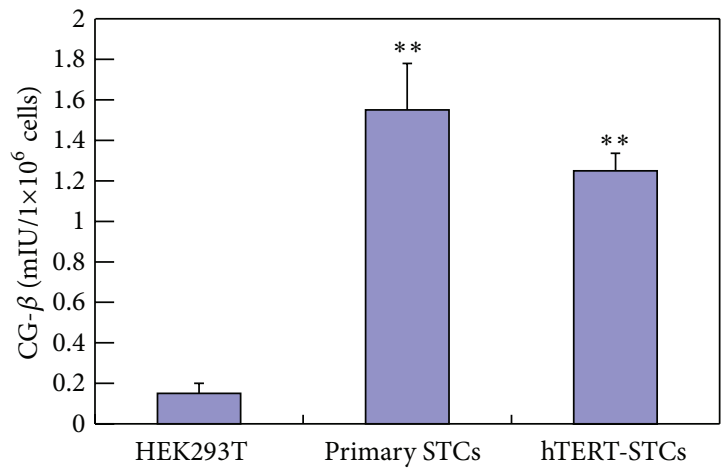

(b)

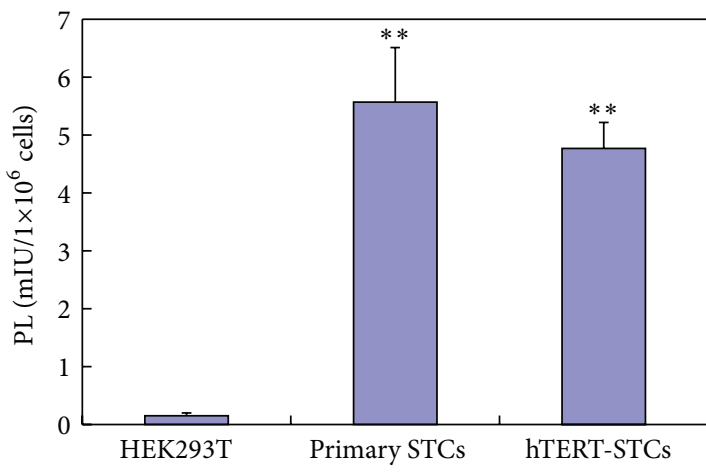

(c)

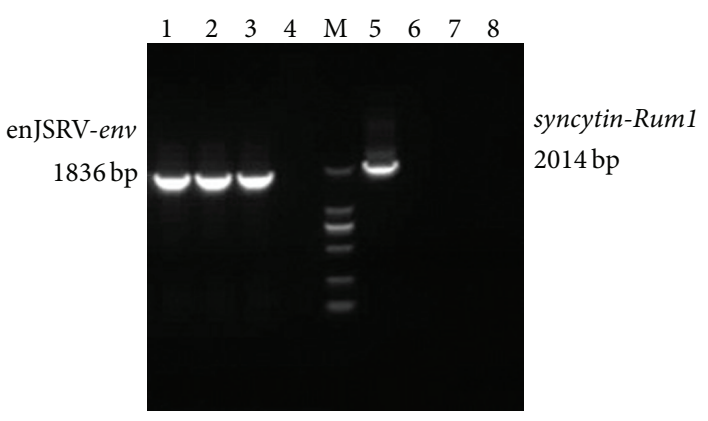

(d)

Figure 3: The biological characteristics of immortalized STCs were found to be similar to that of primary STCs. (a) Immunofluorescence staining of primary STCs for cell keratin 7 expression (1) and hTERT-STCs at passage 50 (2) was positive. Immunofluorescence staining of hTERT-STCs for PBS was (3) (scale bars, $50 \mu \mathrm{m}$ ). (b) The secretion level of primary STCs and hTERT-STCs at passage 50 CG- $\beta$, HEK293T cells (negative control) $\left({ }^{* *} P<0.01\right)$. (c) The secretion level of primary STCs and hTERT-STCs at passage 50 PL, HEK293T cells (negative control) $\left({ }^{* *} P<0.01\right)$. (d) The expression of enJSRV-env and syncytin-Ruml was detected by RT-PCR. Lane 1 was Mongolian sheep genomic DNA; lane 2 was primary STCs; lane 3 was hTERT-STCs at passage 50; and lane 4 was negative control. Lanes 5-8 are the same as in lanes 1-4. hTERT-STCs: human telomerase reverse transcriptase-sheep trophoblast cell line. RT-PCR: reverse transcriptase polymerase chain reaction.

culture of primary STCs not only is time consuming, but is also affected by availability of placenta. Moreover, there may be differences between different batches of cells by virtue of being in different physiological states. To overcome this limitation, there was a need to establish immortal STCs cell lines in vitro.

Immortalization is often achieved by using some viral genes, such as simian virus 40 large $\mathrm{T}$ antigen (SV40 Tag), human papilloma virus E6/E7 genes, and Epstein-Barr virus (EBV) of membrane protein genes, and by using nonviral hTERT gene. By expressing hTERT in human somatic cells, telomerase activity could be reconstructed and a variety of cell lines are established. The sequence and structure of mammalian telomerase RNA were highly conserved, and nucleotide sequence was the same as that of the reverse template. It follows that, in other mammalian cells, expression of hTERT could be combined with telomerase RNA component to reconstruct the functionality of telomerase. Many immortalized mammalian cell lines have been established using this method $[6,26]$.

In this study, hTERT gene was transduced onto STCs to obtain stable cell line hTERT-STCs which can be maintained for 50 generations. We observed continuous expression of TERT gene as indicated by RT-PCR and Western blotting results. Thus the cell line appeared to conserve the telomerase activity. These cells showed monolayer form of growth typical 


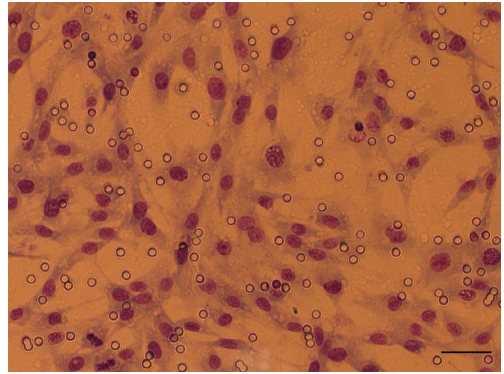

(a)

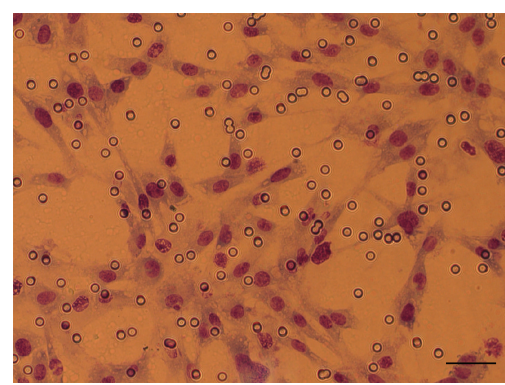

(b)

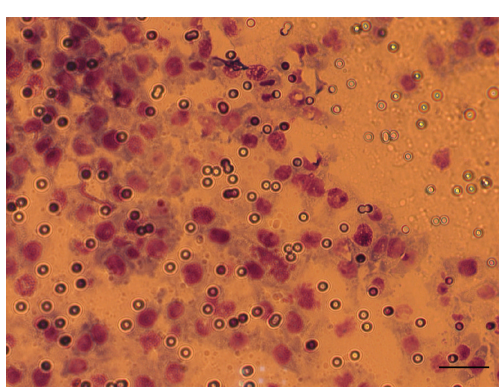

(c)

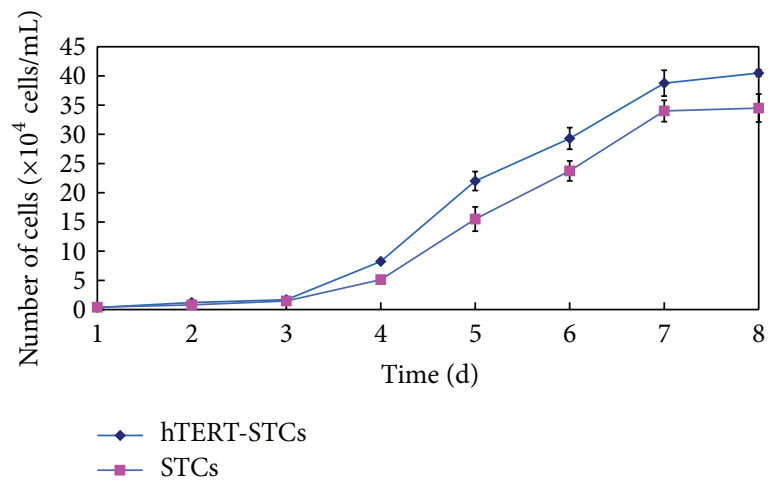

(d)

FIGURE 4: Growth characteristics and invasiveness of primary STCs and immortalized STCs. Primary STCs (a) and hTERT-STCs at passage 50 (b) are seen invading the lower portion of the chamber on Giemsa staining. HeLa cells (c) served as a positive control (scale bars, $50 \mu \mathrm{m}$ ). (d) Growth curves of primary STCs and hTERT-GTCs at passage 50. hTERT-STCs: human telomerase reverse transcriptase-sheep trophoblast cell line.

of cobblestone morphology, and cells were amenable to stable culture. The results of cell growth curve showed that the growth rate of hTERT-STCs was similar to that of primary STCs.

A study comparing the biological characteristics of immortalized and primary sheep trophoblast cells found that the former expressed trophoblast markers such as protein CK7 and demonstrated immortalized STCs with unaltered characteristics of its epithelial origin. At the same time, immortalized sheep trophoblast cells secreted chorionic gonadotrophin (CG) and placental lactogen (PL), although there was no significant difference as compared to primary STCs $(P>0.05)$. Cell invasive studies showed that immortalized sheep trophoblast cells maintained the invasive characteristics of primary STCs. In addition, Cornelis et al. [27] found an envelope gene from the genome of sheep and cattle such that specific expression in the Ovis aries and the Bos taurus in the trophectoderm cells of the conceptus was termed syncytin-Ruml. Of note, the expression of enJSRV-env gene was detected in immortalized sheep trophoblast cells and primary ovine trophoblast cells, but not in syncytin-Ruml. EnJSRV-env largely expressed in the sheep placenta trophectoderm cells in a temporal fashion that corresponded with critical points in onset of trophoblast giant BNC differentiation and conceptus elongation [9, 23, 28]. Therefore immortalized sheep trophoblast cells provide a unique model to study the biological role of enJSRV-env in the development and differentiation of the sheep placenta.
The detection of the transformation ability for immortalized sheep trophoblast cells in vitro and in vivo indicated that they behaved essentially as normal cells without significant transformation ability. They could not grow in the soft agar, did not exhibit any tumorigenicity, and retained the contact inhibition property. These findings attest to the maintenance of biological attributes of primary STCs in hTERT-STCs.

\section{Conclusion}

In conclusion, we established a strain of immortalized sheep trophoblast cell line which retained the basic biological characteristics of primary ovine trophoblast with no potential for malignant transformation. This cell line could be invaluable as an experimental model to study the functionality of trophoblast cells such as hormone secretion, invasiveness, and probable biological function of enJSRV envelope genes.

\section{Conflict of Interests}

The authors declare that there is no conflict of interests regarding the publication of this paper.

\section{Authors' Contribution}

Yufei Zhang and Jing Shi contributed equally to this work. 


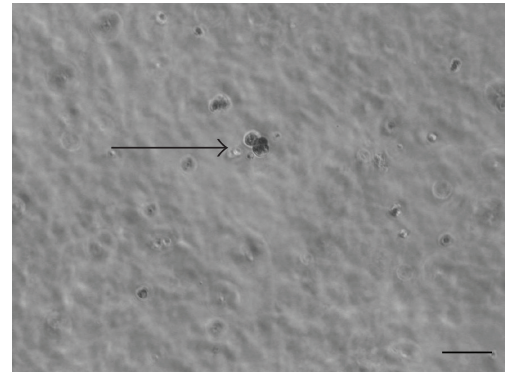

(a)

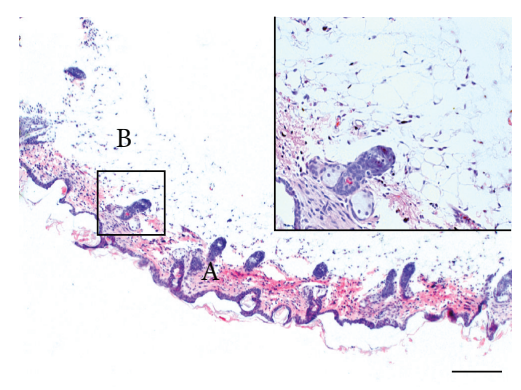

(c)

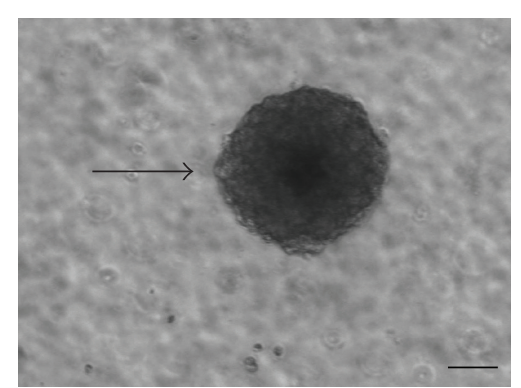

(b)

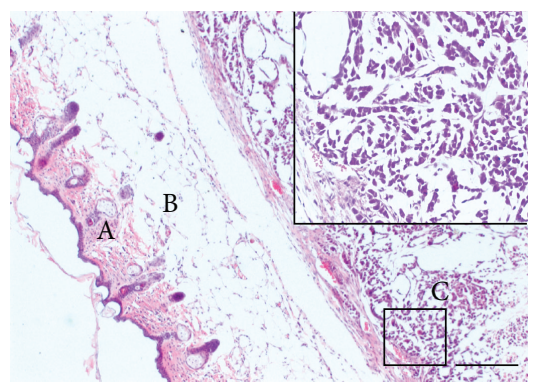

(d)

FIGURE 5: HTERT-STCs did not undergo malignant transformation. (a) hTERT-STCs did not form cell colonies after $>2$ weeks of culture (scale bar, $50 \mu \mathrm{m}$ ). (b) HeLa cells which served as positive control formed cell colonies in the same cultivation time (scale bar, $50 \mu \mathrm{m})$. (c) The organizational structure of nude mice that were injected with hTERT-STCs showing no abnormal cell proliferation at the vaccination site after 2 months. Epidermis and dermis of the skin of the injection site in nude mice (A). Normal subcutaneous connective tissue (B) (scale bar, $25 \mu \mathrm{m}$ ). The selected area is enlarged 5 times in small panels. (d) The organizational structure of nude mice that were injected with HeLa cells, showing a large number of abnormally proliferating tumor cells at the vaccination site after 3 weeks. Epidermis and dermis of the skin of the injection site in nude mice (A). Normal subcutaneous connective tissue (B). Subcutaneous connective tissue with a large number of proliferating tumor cells (C) (scale bar, $25 \mu \mathrm{m}$ ). The selected area is enlarged 5 times in small panel. hTERT-STCs: human telomerase reverse transcriptase-sheep trophoblast cell line.

\section{Acknowledgments}

This report was funded by the National Natural Science Foundation of China (31360597 and 31160493), the Doctor Fund Project of Ministry of Education (20111515110008), and Inner Mongolia Technology Application Research Project (20130224). The pCI-neo-hTERT plasmid was kindly provided by D. Tong (College of Veterinary Medicine, Northwest $A$ and $F$ University, China).

\section{References}

[1] C. Henke, M. Ruebner, F. Faschingbauer et al., "Regulation of murine placentogenesis by the retroviral genes Syncytin-A, Syncytin-B and Peg10," Differentiation, vol. 85, no. 4-5, pp. 150160, 2013.

[2] T. Takao, K. Asanoma, K. Kato et al., "Isolation and characterization of human trophoblast side-population (SP) cells in primary villous cytotrophoblasts and HTR-8/SVneo cell line," PLoS ONE, vol. 6, no. 7, Article ID e21990, 2011.

[3] J. D. Aplin, "Developmental cell biology of human villous trophoblast: current research problems," The International Journal of Developmental Biology, vol. 54, no. 2-3, pp. 323-329, 2010.

[4] M. Knöfler and J. Pollheimer, "Human placental trophoblast invasion and differentiation: a particular focus on Wnt signaling," Frontiers in Genetics, vol. 4, article 190, 14 pages, 2013.
[5] A. S. Bansal, S. A. Bora, S. Saso, J. R. Smith, M. R. Johnson, and M.-Y. Thum, "Mechanism of human chorionic gonadotrophinmediated immunomodulation in pregnancy," Expert Review of Clinical Immunology, vol. 8, no. 8, pp. 747-753, 2012.

[6] F. Dong, Y. Huang, W. Li et al., "The isolation and characterization of a telomerase immortalized goat trophoblast cell line," Placenta, vol. 34, no. 12, pp. 1243-1250, 2013.

[7] A. Tarrade, R. Lai Kuen, A. Malassiné et al., "Characterization of human villous and extravillous trophoblasts isolated from first trimester placenta," Laboratory Investigation, vol. 81, no. 9, pp. 1199-1211, 2001.

[8] Y. Nakaya, K. Koshi, S. Nakagawa, K. Hashizume, and T. Miyazawa, "Fematrin-1 is involved in fetomaternal cell-to-cell fusion in bovinae placenta and has contributed to diversity of ruminant placentation," Journal of Virology, vol. 87, no. 19, pp. 10563-10572, 2013.

[9] S. G. Black, F. Arnaud, M. Palmarini, and T. E. Spencer, "Endogenous retroviruses in trophoblast differentiation and placental development," American Journal of Reproductive Immunology, vol. 64, no. 4, pp. 255-264, 2010.

[10] F. B. P. Wooding, "The synepitheliochorial placenta of ruminants: binucleate cell fusions and hormone production," Placenta, vol. 13, no. 2, pp. 101-113, 1992.

[11] U. M. Igwebuike, "Trophoblast cells of ruminant placentas-a minireview," Animal Reproduction Science, vol. 93, no. 3-4, pp. 185-198, 2006. 
[12] J. Hustin, E. Jauniaux, and J. P. Schaaps, "Histological study of the materno-embryonic interface in spontaneous abortion," Placenta, vol. 11, no. 6, pp. 477-486, 1990.

[13] J. Pollheimer and M. Knöfler, "The role of the invasive, placental trophoblast in human pregnancy," Wiener Medizinische Wochenschrift, vol. 162, no. 9, pp. 187-190, 2012.

[14] A. King, L. Thomas, and P. Bischof, "Cell culture models of trophoblast II: trophoblast cell lines-a workshop report," Placenta, vol. 21, pp. S113-S119, 2000.

[15] W. C. Hahn, S. A. Stewart, M. W. Brooks et al., "Inhibition of telomerase limits the growth of human cancer cells," Nature Medicine, vol. 5, no. 10, pp. 1164-1170, 1999.

[16] H. E. Moon, S. H. Yoon, Y. S. Hur et al., "Mitochondrial dysfunction of immortalized human adipose tissue-derived mesenchymal stromal cells from patients with Parkinson's disease," Experimental Neurobiology, vol. 22, no. 4, pp. 283-300, 2013.

[17] E. H. Blackburn, "Structure and function of telomeres," Nature, vol. 350, no. 6319, pp. 569-573, 1991.

[18] C. E. Moffatt-Jauregui, B. Robinson, A. V. de Moya et al., "Establishment and characterization of a telomerase immortalized human gingival epithelial cell line," Journal of Periodontal Research, vol. 48, no. 6, pp. 713-721, 2013.

[19] L. Balducci, A. Blasi, M. Saldarelli et al., "Immortalization of human adipose-derived stromal cells: production of cell lines with high growth rate, mesenchymal marker expression and capability to secrete high levels of angiogenic factors," Stem Cell Research \& Therapy, vol. 5, no. 3, article 63, pp. 1086-1186, 2014.

[20] M. C. Smith, E. T. Goddard, M. Perusina Lanfranca, and D. J. Davido, "hTERT extends the life of human fibroblasts without compromising type I interferon signaling," PLoS ONE, vol. 8, no. 3, Article ID e58233, pp. 58233-58244, 2013.

[21] H. Bai, T. Sakurai, R. Bai et al., "Establishment and characterization of immortalized bovine endometrial epithelial cells," Animal Science Journal, vol. 85, no. 8, pp. 799-804, 2014.

[22] M. G. Petroff, T. A. Phillips, H. Ka, J. L. Pace, and J. S. Hunt, "Isolation and culture of term human trophoblast cells," Methods in Molecular Medicine, vol. 121, pp. 203-217, 2006.

[23] K. A. Dunlap, M. Palmarini, D. L. Adelson, and T. E. Spencer, "Sheep endogenous betaretroviruses (enJSRVs) and the hyaluronidase 2 (HYAL2) receptor in the ovine uterus and conceptus," Biology of Reproduction, vol. 73, no. 2, pp. 271-279, 2005.

[24] K. A. Dunlap, M. Palmarini, M. Varela et al., "Endogenous retroviruses regulate periimplantation placental growth and differentiation," Proceedings of the National Academy of Sciences of the United States of America, vol. 103, no. 39, pp. 14390-14395, 2006.

[25] T. E. Spencer and M. Palmarini, "Application of next generation sequencing in mammalian embryogenomics: lessons learned from endogenous betaretroviruses of sheep," Animal Reproduction Science, vol. 134, no. 1-2, pp. 95-103, 2012.

[26] H. X. Hong, Y. M. Zhang, H. Xu, Z. Y. Su, and P. Sun, "Immortalization of swine umbilical vein endothelial cells with human telomerase reverse transcriptase," Molecules and Cells, vol. 24, no. 3, pp. 358-363, 2007.

[27] G. Cornelis, O. Heidmann, S. A. Degrelle et al., "Captured retroviral envelope syncytin gene associated with the unique placental structure of higher ruminants," Proceedings of the National Academy of Sciences of the United States of America, vol. 110, no. 9, pp. E828-E837, 2013.
[28] Y. F. Zhang, J. Shi, and S. Y. Liu, "Recent advances in the study of active endogenous retrovirus envelope glycoproteins in the mammalian placenta," Virologica Sinica, vol. 30, no. 4, pp. 239248, 2015. 

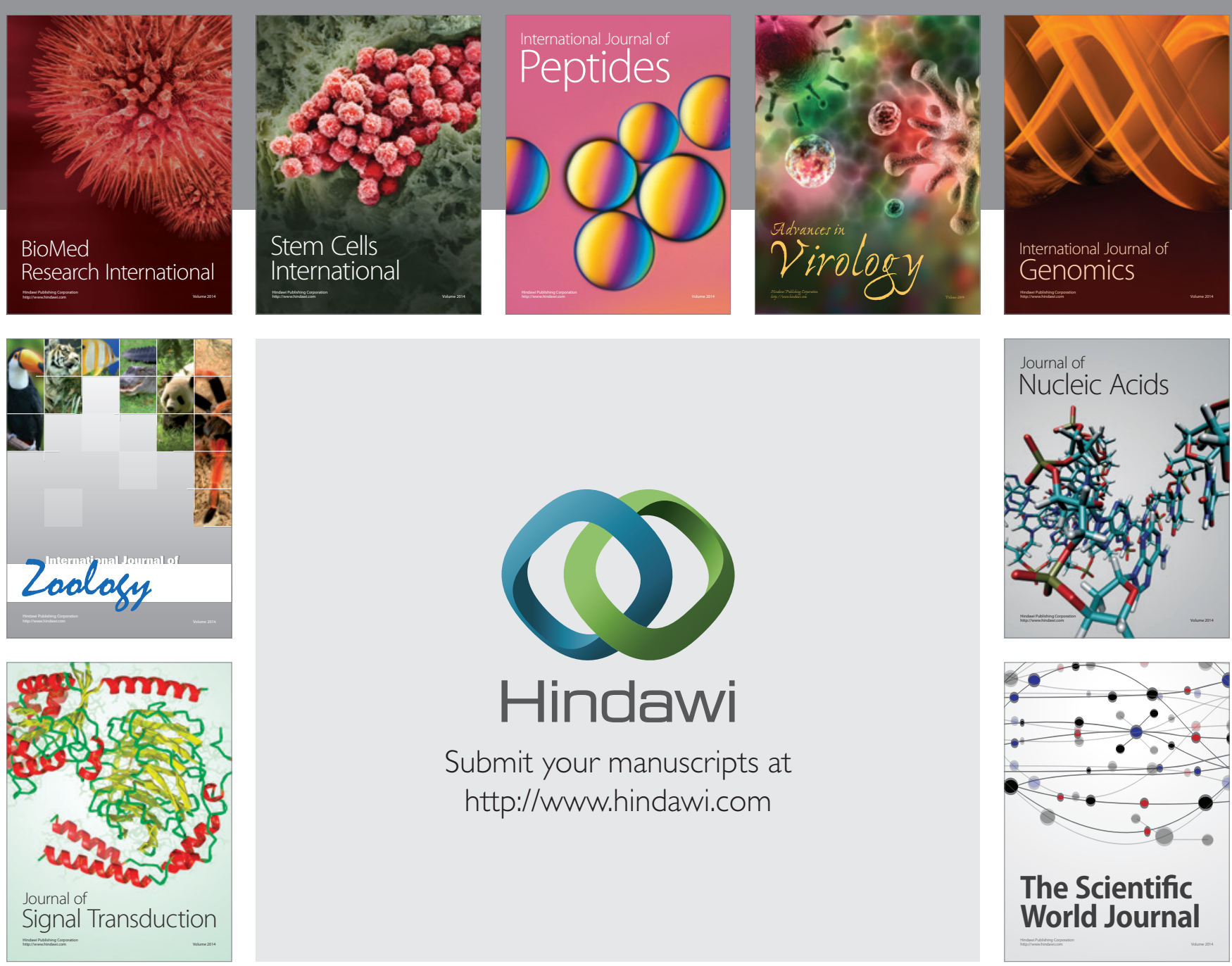

Submit your manuscripts at

http://www.hindawi.com
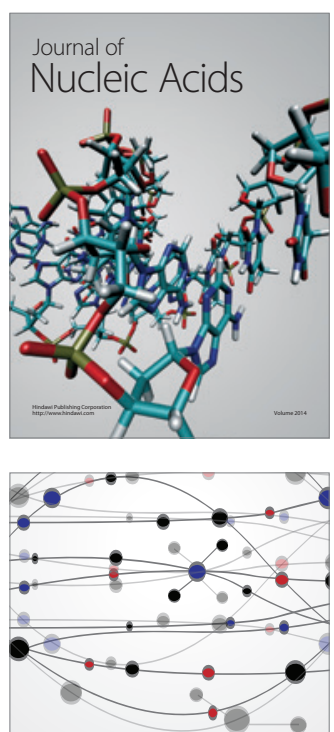

The Scientific World Journal
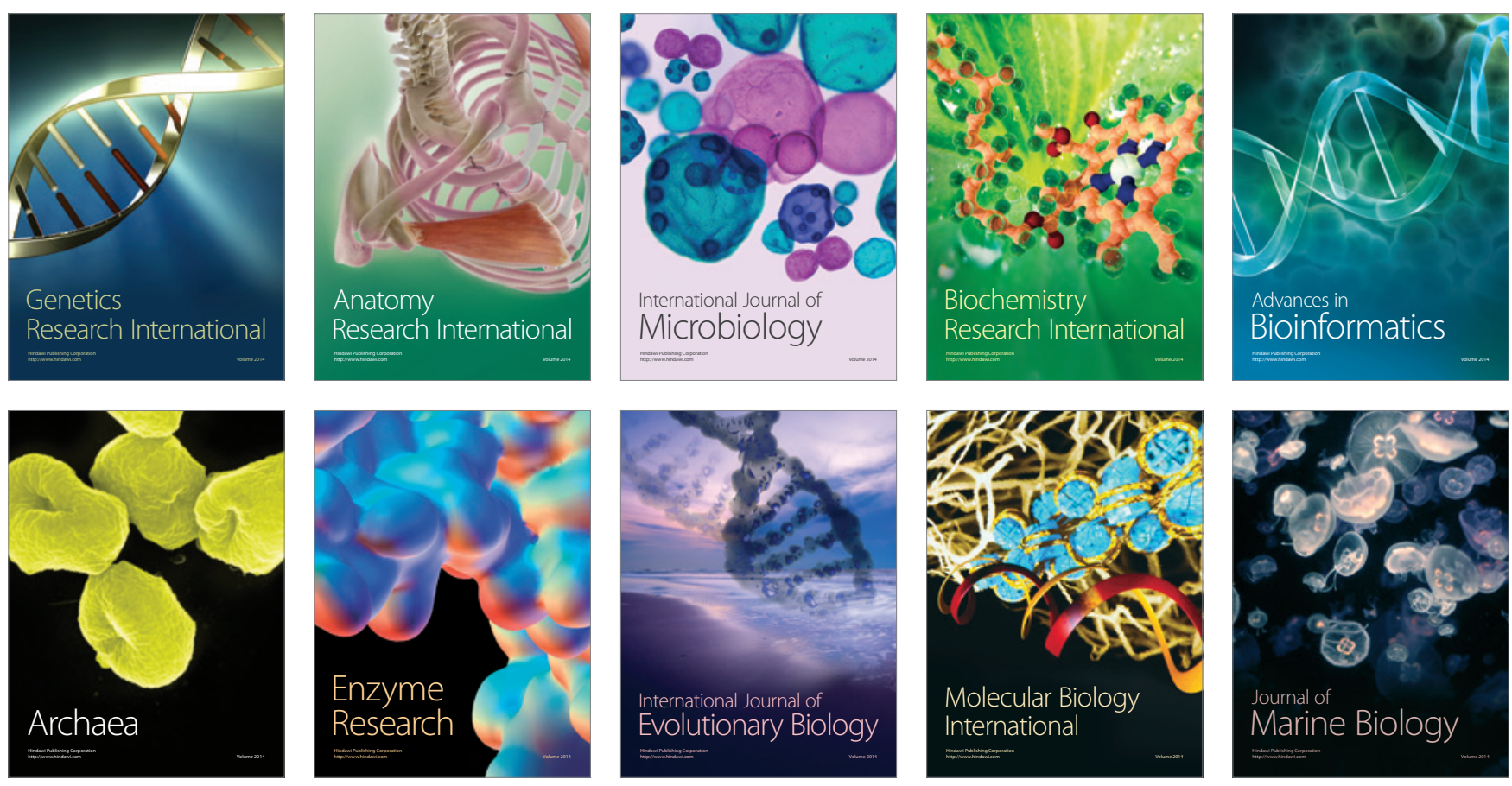